\title{
THE ROLE OF SYNERGY AND COMPLEMENTARITY IN A MULTICHANNEL E-COMMERCE SYSTEM
}

\author{
Gerhard Wagner, University of Siegen, Germany \\ Hanna Schramm-Klein, University of Siegen, Germany \\ Sascha Steinmann, University of Siegen, Germany
}

\begin{abstract}
When online retailers operate more than one electronic distribution channel (e-channel), they have to manage the individual online distribution channels (such as an online Web site and a mobile shopping app), but they also have to manage the degree of synergies and complementarity across these channels. Though many retailers already provide diverse online shopping channels, there is no research that investigated the role of synergies and complementarity across these e-channels as well as their effects on consumers' intention to use a retailer's multichannel e-commerce system.

Most studies on consumer behavior in e-commerce are limited to customers visiting and purchasing on a retailer's Web site (e.g. Liu and Forsythe 2011; Ha and Stoel 2009) or consumers' intentions to shop online in general (e.g. Liu, Forsythe, and Black 2011). However, only few authors indicate that e-commerce includes a wider range of online shopping possibilities. The perspective of multiple e-commerce channels is supported by Zhang et al. (2010) who anticipate that new digital channel formats will develop and alter the retail landscape. We agree and indicate that this development might induce that online pure-players, such as Amazon.com, evolve into multichannel e-commerce retailers. While the role of synergies and complementarity in traditional multichannel environments (e.g. retail store and catalog) has received wide attention (e.g. Kollmann, Kuckertz, and Kayser 2012; Avery et al. 2007; Montoya-Weiss, Voss, and Grewal 2003), the findings and implications are only to some extent transferable to an environment of multiple e-channels.
\end{abstract}

This study is multidisciplinary and contributes to research in multichannel retailing and e-commerce by investigating the role of synergy and complementarity across electronic distribution channels and their effects on consumer behavior. Based on diffusion, information integration and resource-based theory we develop a conceptual framework for a multichannel ecommerce environment and empirically test it with a data set of $N=904$ consumers.

Our findings underline the relevance of cross-channel synergies and complementarity in multichannel e-commerce systems. The results of our empirical study suggest that synergies and complementarity are relevant antecedents of consumer behavior, particularly the intention to use a retailer's multichannel e-commerce system. When customers use diverse Internet-enabled devices for online shopping the interaction of these different e-channels is of relevance to build a well integrated multichannel e-commerce system. Moreover, our findings emphasize the role of complementarity across e-channels, in terms of heterogeneity across individual e-channels. With other words, an additional e-channel only creates enhanced value if it offers extra benefit. Retailers can use this knowledge to create a "seamless" e-channel environment and underline the individual benefits of single e-channels.

References available upon request. 